\title{
Characteristics of the oestrous cycle and influence of social factors in grey short-tailed opossums (Monodelphis domestica)
}

\author{
B. H. Fadem and R. S. Rayve \\ Department of Psychiatry and Mental Health Science, University of Medicine and Dentistry of New \\ Jersey, New Jersey Medical School, 100 Bergen Street, Newark, New Jersey 07103, U.S.A.
}

\begin{abstract}
Summary. Characteristics of the oestrous cycle of grey short-tailed opossums were studied by vaginal smears. The period of oestrus was identified by a sudden proliferation of epithelial cells which lasted about 6 days (range 3-12 days), followed by a leucocytic infiltration. Oestrous cycle length showed a bimodal distribution of 14.4 days ( 5 cycles, range $11-17$ days) and $32 \cdot 3$ days ( 10 cycles, range $28-39$ days). Females housed with males showed more days of epithelial cell proliferation than did females housed alone, and oestrous periods tended to occur in synchrony, suggesting that social factors may influence the oestrous cycle in this species.
\end{abstract}

\section{Introduction}

The grey short-tailed opossum (Monodelphis domestica) is an American opossum of the Family Didelphidae which includes the common North American opossum (Didelphis virginiana) and the mouse opossum (Marmosa robinsoni). This is the most ancient marsupial family from which all other New World and Australian marsupials appear to be derived (Clemens, 1968; Keast, 1977). The common opossum and the mouse opossum have been proposed as marsupial species for use in experimental biology (Barnes, 1968; Jurgelski, 1974), but have proved impractical mainly because of breeding difficulties.

We report here on the characteristics of the oestrous cycle in grey short-tailed opossums because (1) there are few reports of the natural biology of this species (McNab, 1978; Streilein, 1982; Christian, 1983), (2) laboratory breeding has been obtained through 7 generations, indicating the usefulness of this animal for biomedical research (Fadem, Trupin, VandeBerg, Maliniak \& Hayssen 1982; VandeBerg, 1983), and (3) there is evidence that social factors influence breeding in this opossum, i.e. animals become reproductively inactive if housed alone for extended periods (Maliniak \& Taft, 1981) and breeding is stimulated if males in breeding pairs are changed (Fadem et al., 1982).

\section{Materials and Methods}

Animals. The grey short-tailed opossum (Monodelphis domestica) is found in the arid Chaco, Cerrado and Caatinga regions and in the remnant Atlantic rainforest on the eastern border of the Caatinga region in Brazil (Streilein, 1982). The animals used in the present study were part of a breeding colony at the University of Medicine and Dentistry of New Jersey, New Jersey Medical School, Newark, and were descendants of 9 animals trapped in the Caatinga region in 1978 by representatives of the National Zoological Park in Washington, D.C.

Adult female grey opossums weigh $80-100 \mathrm{~g}$ and adult males $90-150 \mathrm{~g}$. Head and body length of 
adults ranges from 170 to $200 \mathrm{~mm}$ with tail length an additional $60-80 \mathrm{~mm}$. The coat colour is greybrown with lighter underparts. Sexual maturity is reached at 4-5 months; reproduction has occurred up to 28 months of age in females and 39 months in males, and breeding occurs throughout the year. Individual females have had up to 4 litters per year (VandeBerg, 1983). The gestation period is 14-15 days. Most litters contain between 5 and 12 offspring and post-partum dependence is about 50 days (Maliniak \& Taft, 1981 ; Fadem et al., 1982; VandeBerg, 1983). Thirteen females aged 10-24 months were used in the present study.

Maintenance. All animals were housed in one $3.4 \times 6.1 \mathrm{~m}$ room in the animal facility at the New Jersey Medical School, Newark, for a minimum of 4 months before the start of the study. A reversed lighting schedule was used with white fluorescent light for $14 \mathrm{~h}(21: 00-11: 00 \mathrm{~h}) / 24 \mathrm{~h}$ and temperature was maintained at $24^{\circ} \mathrm{C}$. Water and food were available ad libitum. Complete details on the laboratory maintenance of these animals appear elsewhere (Maliniak \& Taft, 1981; Fadem et al., 1982; VandeBerg, 1983).

To look at the effect of social factors on the oestrous cycle, individual females were housed (1) alone in a polyethylene rat cage $(46 \times 23 \times 19 \mathrm{~cm})$ with a wire-mesh top, placed on a cage rack spatially separated by at least $2 \mathrm{~m}$ from the males in the colony $(\mathrm{N}=4)$; (2) with a male in a stainless-steel cage $(100 \times 80 \times 75 \mathrm{~cm})(\mathrm{N}=6)$; or (3) inside a plastic rat cage (as in 1) placed inside a stainless-steel cage (as in 2) in which a male was allowed free movement $(\mathrm{N}=6)$. The condition for Group 3 was devised to permit sensory contact between male and female while preventing mating and hence pregnancy from occurring. Females in Groups 2 and 3 were housed as indicated for at least 2 weeks before the beginning of the study.

Vaginal smears. Vaginal smears were obtained by lavage of the urogenital sinus with $0.1 \mathrm{ml}$ saline $(9 \mathrm{~g} \mathrm{NaCl} / 1)$ using a glass medicine dropper. To obtain the smear, the dropper was inserted approximately $5 \mathrm{~mm}$ into the urogenital sinus, aimed slightly downward and the saline injected with gentle pressure on the dropper bulb. Finger pressure on the bulb was released as the tube was withdrawn. During this procedure, the animal grasped a wire mesh with the forepaws and the body was held up at $45^{\circ}$ angle by the tail. The aspirated fluid was spread on an albumin-coated slide, dried in a warming oven at $30^{\circ} \mathrm{C}$ for $10 \mathrm{~min}$ until the fluid evaporated, and then fixed in $95 \%$ ethanol for $20 \mathrm{~min}$ before staining. The smears were stained with the Papanicolaou technique (Papanicolaou, 1954), using Lillie-Meyer haematoxylin and commercially available stains (Fisher Scientific Corporation, Springfield, New Jersey). The smears were then mounted with Permount.

Identification of oestrus. To identify the vaginal cell changes typical of oestrus in grey short-tailed opossums, the vaginal smear records of 3 females that subsequently gave birth and of 2 females that were observed mating were examined before the start of the present study. In marked contrast to previous smears taken from these 5 animals, smears taken at oestrus contained large numbers of epithelial cells. The three major cell types recognized in these smears were (1) basophilic, nucleated, intermediate cells with an elongated form and folded borders; (2) acidophilic, nucleated and nonnucleated superficial cells with a polygonal outline and (3) leucocytes. Basophilic nucleated and non-nucleated superficial cells and small basophilic parabasal cells were also seen. A proliferation of cells for 3 or more days followed by leucocytic infiltration became the criterion for identification of oestrus for the present study.

The two females that were observed mating did so on the second day of the period of cellular proliferation. Female grey short-tailed opossums have, however, been seen to mate on two consecutive nights, and on one occasion a second copulation occurred $34 \mathrm{~h}$ after the first (Trupin \& Fadem, 1982). Thus, although the term 'oestrus' is used here to identify the period of cellular proliferation, the actual start of behavioural oestrus has not been pinpointed in this species. Using the 14-15-day gestation period as a reference point, the 2 nd and 3 rd days of the period of cellular proliferation were identified as the probable days of conception for the females that had litters.

Vaginal smearing schedule. Vaginal smears were taken on two occasions and 650 vaginal smears were obtained. On the first occasion, 6 females were each housed with a male (Group 2) and smears 


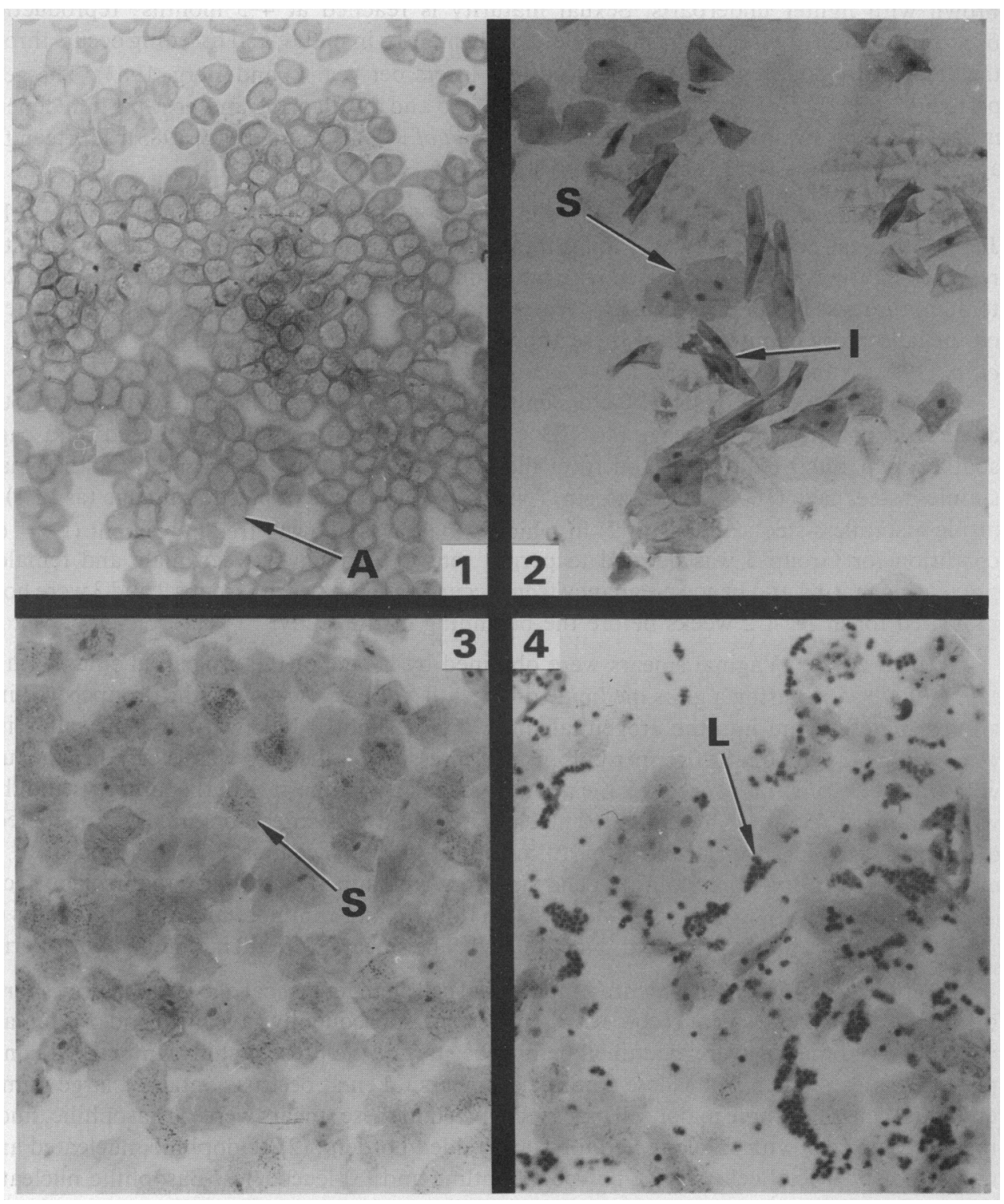

Fig. 1. Vaginal smear containing anal gland secretion (A).

Fig. 2. Smear taken on the first day of the period of oestrus. Note the basophilic intermediate cells (I) and superficial cells (S).

Fig. 3. Smear taken on the fourth day of the oestrous period. Note the preponderance of anucleated, granular, acidophilic superficial cells (S).

Fig. 4. Smear taken on the sixth day of the oestrous period. Leucocytic infiltration has occurred (L) although some epithelial cells remain. 
were taken daily 6 days per week for a total of 51 days. On the second occasion, 2 months after the end of the period, 10 females ( 4 in Group 1, 6 in Group 3) were used and vaginal smears were taken daily, 6 days per week for a total of 47 days.

Data analyses. The following measures were derived: (1) 'many-cell' days (days on which a proliferation of epithelial cells was seen in the vaginal smear such that at least $50 \%$ of the microscope field was covered by cells), (2) 'few-cell' days (days on which this proliferation of epithelial cells was not seen), (3) period of oestrus ( 3 or more consecutive 'many-cell' days), (4) oestrous cycle length (number of days from the first day of one period of oestrus to the first day of the next period of oestrus, and (5) ratio (number of 'many-cell' days divided by the number of 'fewcell' days in the smearing period).

Differences between the 3 groups were analysed by using a nested analysis of variance (ANOVA) design with animals nested within housing condition (repeated measures design). Since preliminary analyses indicated no statistically significant differences between Groups 2 and 3, the data for these groups were pooled for subsequent analyses. Means are reported with standard deviations.

To test statistically for synchrony of oestrus, a window of specified width was placed over contiguous data for all animals on each of the two occasions ( 6 animals on the first occasion and 10 animals on the second occasion). The average number of animals in maximal agreement per day, i.e. those in oestrus or those not in oestrus (whichever was larger), was calculated over all days within the window. This statistic, $w$, was taken as the characteristic of the data set. To generate the empirical distribution, the oestrous record of each animal was shifted both ahead and backward, always maintaining a full window of data, and w was calculated for each shifting. This procedure was repeated for the data set with windows of varied width and placement (for each test, the width of the window was constant). The significance level of the different window conditions was then averaged. By comparing $w$ of the observed data to the empirical distribution, the significance level was determined.

\section{Results}

\section{Cyclic changes in the vaginal smear cell pattern}

During dioestrus, the vaginal smears contained only a few scattered epithelial cells, both basophilic and acidophilic. Leucocytes in small numbers were occasionally present. Occasionally, a smear contained many small circular basophilic structures (PI. 1, Fig. 1). This is likely to be a secretion of the anal glands which are present in didelphids (Jurgelski \& Porter, 1974). On the 1st and 2 nd days of the period of oestrus, the smear was characterized by a marked increase in the number of epithelial cells. Approximately $60 \%$ of these cells were basophilic intermediate cells and $40 \%$ were basophilic and acidophilic superficial cells. Some basophilic parabasal cells were also seen (Pl. 1, Fig. 2). On Days 3-5 of the period of oestrus, 95\% of the cells in the smear were anucleated, granular, acidophilic, superficial cells (Pl. 1, Fig. 3). On Days 6-8 after the start of the period of oestrus, the smear was characterized by a leucocytic infiltration (P1. 1, Fig. 4), and by Day 8 , few epithelial cells remained. In all but one animal, a proliferation of epithelial cells was also seen one to three times during each period of dioestrus. These proliferations of 'many-cell' days usually lasted 1 or occasionally 2 days, and were not followed by a leucocyte infiltration.

\section{Characteristics of the oestrous cycle}

Of the 13 females from which vaginal smears were taken, 2 were represented in Groups 2 and 3 and 1 in Groups 1 and 2 . In 15 of the 16 smearing periods for the 13 females, at least one period of oestrus was seen. One female in Group 1 failed to exhibit a period of oestrus although she had shown two periods of oestrus when housed with a male (Group 2). Average length of the period of oestrus was $5 \cdot 9$ days (s.d. $2 \cdot 1$ days) and average oestrous cycle length was $26 \cdot 3$ days (s.d. $9 \cdot 2$ days). 
Part of the variability in oestrous cycle length was due to the fact that this measure was bimodally distributed with no overlap between the two groups. In 5 cycles, the mean \pm s.d. oestrous cycle length was $14.4 \pm 2.8$ days and ranged from 11 to 17 days, while in the remaining 10 cycles, the mean oestrous cycle length was 32.3 days \pm 3.4 and ranged from 28 to 39 days (Text-fig. 1). Two females exhibited two short cycles each and one female exhibited both one long and one short cycle.

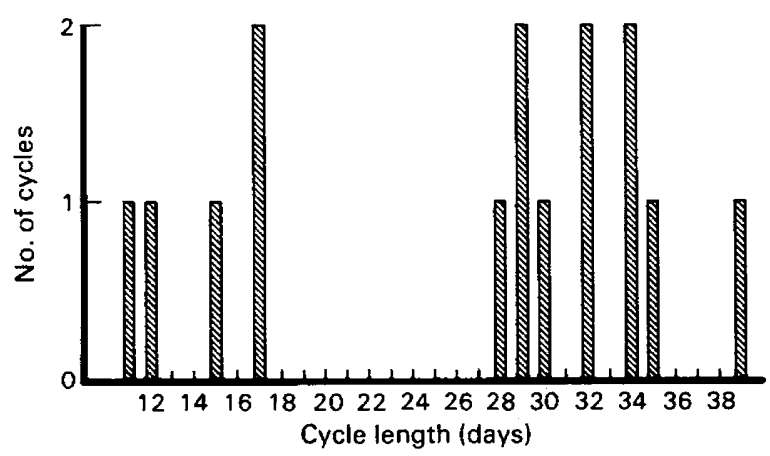

Text-fig. 1. Bimodal distribution of oestrous cycle length in the grey short-tailed opossum (Monodelphis domestica).

The length of the period of oestrus did not differ between long and short cycles. Although there were no significant differences in the duration of the period of oestrus or oestrous cycle length amongst the 3 groups, all of the shorter cycles occurred in Group 3. Although there was also no significant difference in duration of oestrus amongst the groups, females in Group 1 showed significantly fewer 'many-cell' days, both during and between periods of oestrus, than did females living with or exposed to males (Groups 2 and 3$)(\mathrm{F}=8.75$, $\mathrm{df}=1,11 ; P<0.01)$. In the present study, the occurrence of oestrus tended to be synchronous $(P<0.01)$ (Text-fig. 2).

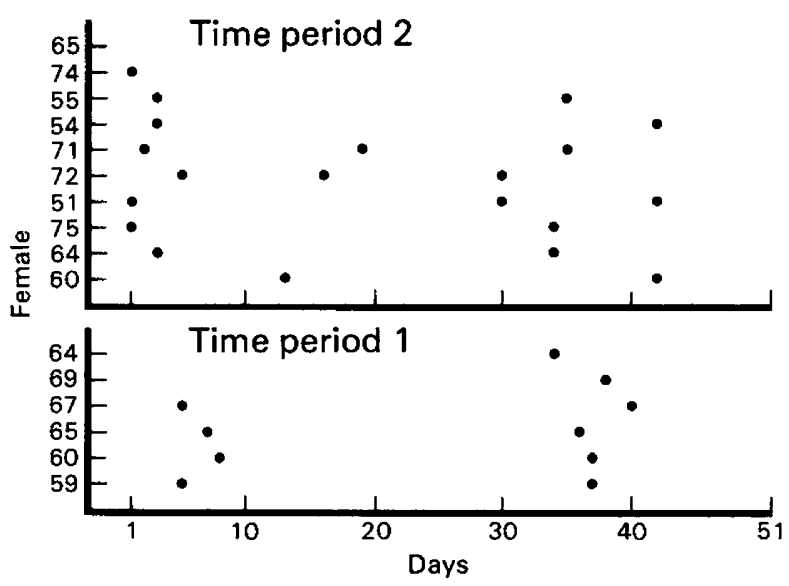

Text-fig. 2. Synchrony of oestrus in the grey short-tailed opossum (Monodelphis domestica). Each symbol represents the first day of a period of oestrus. 


\section{Discussion}

The vaginal cytological changes seen here in the oestrous cycle of the grey short-tailed opossum, were much like those of other polyoestrous marsupials (Sharman, 1955; Pilton \& Sharman, 1962; Jurgelski \& Porter, 1974; Godfrey, 1975; Peters \& Rose, 1979) and of commonly studied eutherians such as the rat (Feder, 1981). This is not unexpected since the hormonal control of the oestrous cycle is similar in marsupials and eutherians (Sharman, 1970; Sutherland, Evans \& Tyndale-Biscoe, 1980).

As in other marsupials (Peters \& Rose, 1979), the period of oestrus of the grey short-tailed opossum was long (range 3-12 days) compared with that of the rat (1 day) (Feder, 1981). In rats, smears are taken directly from the vagina but in this study smears were taken from the posterior vaginal sinus although the most pronounced cornification of the vaginal complex occurs in the lateral vaginae which may be cornified for extended periods during the oestrous cycle. The influx of these cornified cells from the lateral vaginae can mask changes in the cells which line the posterior vaginal sinus resulting in the smears being cornified for an extended period (Peters \& Rose, 1979). This might explain both the extended length of the period of oestrus and the single or double 'manycell' days seen between periods of oestrus in the present study. This characteristic also makes it difficult to predict ovulation and thus the likelihood of mating simply by monitoring vaginal smears.

In the present study, oestrous cycle length showed a bimodal distribution with one group of cycles being about 2 weeks long and another group just over 1 month long. This is in contrast to the oestrous cycles shown by the polyoestrous Australian marsupials Trichosurus vulpecula, Setonix brachyurus and Vombatus ursinus which are all approximately 1 month in length and range in individuals from 22 to 33 days (Sharman, 1955; Pilton \& Sharman, 1962; Peters \& Rose, 1979). The oestrous cycle of the common North American opossum averages 1 month in length (range 17-38 days) (Hartman, 1923; Jurgelski \& Porter, 1974; Fleming \& Harder, 1981). Like the grey shorttailed opossum, the mouse opossum shows an oestrous cycle length with a bimodal distribution: one group of cycles averaging 15.6 days and the other group 26 days in length. In the mouse opossum, the longer cycles are normal ovulatory cycles while the shorter ones represent anovulatory cycles which are not accompanied by behavioural oestrus (Godfrey, 1975).

External-social factors apparently influenced the oestrous cycles of grey short-tailed opossums in the present study. Not only did females show synchronous exhibition of oestrus, but those living in proximity to males were more likely to show vaginal smears typical of oestrus over the course of the oestrous cycle than those that lived alone. Although synchrony of oestrus per se has not been heretofore reported in a marsupial, Reynolds (1952) noted that North American opossum females from the same litters came into oestrus at the same time.

Synchrony of oestrus occurs in a number of eutherian mammals, including man, as a result of social-olfactory stimulation (Parkes \& Bruce, 1961) provided by males (Whitten, 1956) or by other females (McClintock, 1971, 1978). The mechanism for this phenomenon probably involves a trigger of gonadotrophin secretion by olfactory stimulation with subsequent oestradiol release (Bronson \& Desjardins, 1969). Whether the external-social effects seen in this study were due to the presence of males or of other females awaits further study. Whatever the cause of these influences on the oestrous cycle, the finding that social factors affect the reproductive physiology of grey shorttailed opossums is not unexpected because odour cues play an important role in social interaction in this species (Fadem et al., 1982; Trupin \& Fadem, 1982; Fadem \& Cole, 1985).

We thank Dr Humberto Villaverde, Dr Robert Brandes, Mr Frank Bella, Mr Nathaniel Brown, Ms Mary Paprocki and the staff of animal caretakers at the UMDNJ-New Jersey Medical School for help with the care and maintenance of animals; Dr Mark Nicolich of the Department of Statistics, Rutgers University, for conducting the statistical analyses; and Dr Susan Feldman of the 
Department of Anatomy, UMDNJ-New Jersey Medical School, for technical expertise. This study was supported in part by a grant from the Foundation of the University of Medicine and Dentistry of New Jersey and by NIH Grant HD 16018 NICHHD.

\section{References}

Barnes, R.D. (1968) Marmosa mitis, a small marsupial for experimental biology. In Animal Models for Biomedical Research, Publication No. 1594, pp. 88-98. National Academy of Sciences, Washington, D.C.

Bronson, F.H. \& Desjardins, C. (1969) Release of gonadotrophin in ovariectomized mice after exposure to males. J. Endocr. 44, 293-297.

Christian, D.P. (1983) Water balance in Monodelphis domestica (Didelphidae) from the semiarid Caatinga of Brazil. Comp. Biochem. Physiol. 74A, 665-669.

Clemens, W.A. (1968) Origin and early evolution of marsupials. Evolution 22, 1-18.

Fadem, B.H. \& Cole, E. (1985) Scent marking in the grey short-tailed opossum (Monodelphis domestica). Anim. Behav. (in press).

Fadem, B.H., Trupin, G.L., VandeBerg, J.L., Maliniak, E. \& Hayssen, V. (1982) Care and breeding of the grey, short-tailed opossum (Monodelphis domestica). Lab. Anim. Sci. 32, 405-409.

Feder, H.H. (1981) Estrous cyclicity in mammals. In Neuroendocrinology of Reproduction, pp. 279--329. Ed. N. T. Adler. Plenum Press, New York.

Fleming, M.W. \& Harder, J.D. (1981) Uterine histology and reproductive cycles in pregnant and nonpregnant opossums, Didelphis virginiana. J. Reprod. Fert. 63, 21-24.

Godfrey, G. (1975) A study of oestrus and fecundity in a laboratory colony of mouse opossums (Marmosa robinsoni). J. Zool., Lond. 175, 541-555.

Hartman, C.G. (1923) The oestrous cycle in the opossum. Am. J. Anat. 32, 353-421.

Jurgelski, W., Jr (1974) The opossum (Didelphis virginiana Kerr) as a biomedical model. I. Research perspective, husbandry and laboratory techniques for the opossum. Lab. Anim. Sci. 24, 376-403.

Jurgelski, W., Jr \& Porter, M. E. (1974) The opossum (Didelphis virginiana Kerr) as a biomedical model. III. Breeding the opossum in captivity: methods. $L a b$. Anim. Sci. 24, 412-425.

Keast, A. (1977) Historical biogeography of the marsupials. In The Biology of Marsupials, pp. 69-95. Eds. F. Stonehouse \& D. Gilmore. University Park Press, Baltimore.

Maliniak, E. \& Taft, L. (1981) Husbandry and breeding protocol for Monodelphis domestica. Bull. No. I Dept. Zool. Res., pp. 1-4. National Zoological Park, Washington.

McClintock, M.K. (1971) Menstrual synchrony and suppression. Nature, Lond. 22, 224-245.
McClintock, M.K. (1978) Estrous synchrony and its mediation by airborne chemical communication (Rattus norvegicus). Horm. Behav. 10, 267-276.

McNab, B.K. (1978) The comparative energetics of Neotropical marsupials. J. comp. Physiol. 125, 115128.

Papanicolaou, G.N. (1954) Atlas of Exfoliative Cytology. Harvard University Press, Cambridge, Mass.

Parkes, A.S. \& Bruce, H.M. (1961) Olfactory stimuli in mammalian reproduction. Science, N.Y. 134, 10491054.

Peters, D. \& Rose, R. (1979) The oestrous cycle and basal body temperature in the common wombat (Vombatus ursinus). J. Reprod. Fert. 57, 453-460.

Pilton, P. \& Sharman, G. (1962) Reproduction in the marsupial Trichosurus vulpecula. J. Endocr. 25, 119136.

Reynolds, H. C. (1952) Studies on reproduction in the opossum (Didelphis virginiana). Univ. Cal. Publ. Zool. 52, $223-284$.

Sharman, G. (1955) Studies on marsupial reproduction. 2. The oestrous cycle of Setonix brachyurus. Aust. J. Zool. 3, 44-55.

Sharman, G. (1970) Reproductive physiology of marsupials. Science, N.Y. 167, 1221-1228.

Streilein, K.E. (1982) Behavior, ecology, and distribution of South American marsupials. In Mammalian Biology in South America, pp. 231-249. Eds M. A. Mores \& H. H. Genoways. Pymatuning Laboratory of Ecology: Special Publication, Linesville.

Sutherland, R.L., Evans, S.M. \& Tyndale-Biscoe, C.H. (1980) Macropodid marsupial luteinizing hormone: validation of assay procedures and changes in concentrations in plasma during the oestrous cycle in the female tammar wallaby (Macropus eugenii). $J$. Endocr. 86, 1-12.

Trupin, G.L. \& Fadem, B.H. (1982) Sexual behaviour of the gray short-tailed opossum (Monodelphis domestica). J. Mammal. 63, 409-414.

VandeBerg, J.L. (1983) The gray short-tailed opossum: a new laboratory animal. Institute of Laboratory Animal Resources News 26, 9-12.

Whitten, W.K. (1956) Modification of the oestrous cycle of the mouse by external stimuli associated with the male. J. Endocr. 13, 399-404. 